\title{
ANTIBIOTIC UTILIZATION PATTERN AT THE SURGERY DEPARTMENT OF A TERTIARY CARE HOSPITAL
}

\author{
ASAWARI RAUT*, TIRZAH CHERIAN, SHREEPA CHAUHAN, ATMARAM PAWAR \\ Department of Clinical Pharmacy, Poona College of Pharmacy, Bharati Vidyapeeth Deemed University, Pune, Maharashtra, India. \\ Email: drpawarpcp@gmail.com
}

Received: 23 February 2017, Revised and Accepted: 11 March 2017

\section{ABSTRACT}

Objective: This study aims to determine the pattern of antibiotic utilization at the surgery department of a tertiary care hospital.

Methods: A prospective observational study was conducted over a period of 6-month in surgical ward at Bharti Hospital and Research Centre, Pune, a 1000 bedded teaching hospital. Patients above 18 years and receiving antibiotic therapy pre- and post-surgery were included in the study.

Results: A total of 160 patients with surgical operations were included in the study. The disease spectrum was classified into respective system-wise surgical procedures of which $49.37 \%$ cases are skin and soft tissue infections, $25.62 \%$ cases of general surgical procedure, $12.5 \%$ cases of gastrointestinal surgical procedure, $11.25 \%$ cases of urinary system, and $1.25 \%$ case of head-neck system. $20.62 \%$ of the study patients had hernia, $18.12 \%$ patients had cellulitis, $16.87 \%$ patients had diabetic foot ulcer, $16.25 \%$ patients had abscess, and $10.62 \%$ patients had appendicitis and cholelithiasis. In this study, it was found that 471 antibiotics were used in total of 160 patients, among which highest group of antibiotics prescribed were third generation cephalosporin (28.23\%) followed by penicillins (23.56\%). The most frequently prescribed antibiotics were metronidazole - $19.74 \%$ among the nitroimidazoles followed by ceftriaxone $-19.53 \%$ of the class cephalosporins.

Conclusion: The rate of prescribing of broad-spectrum antibiotics has increased demonstrably which may result in the development of bacterial resistance; however, development of guidelines for an antibiotic prescription and use of appropriate drugs for the diseases can minimize the unfavorable use of antibiotics and cost of health care.

Keywords: Antibiotics, Surgery, Prescription pattern.

(c) 2017 The Authors. Published by Innovare Academic Sciences Pvt Ltd. This is an open access article under the CC BY license (http://creativecommons. org/licenses/by/4. 0/) DOI: http://dx.doi.org/10.22159/ajpcr.2017.v10i6.18099

\section{INTRODUCTION}

Surgical site infections (SSIs) are the second most common cause of nosocomial infections in surgical patients. Antibiotics when used prophylactically contribute to the minimization of incidences of SSIs and are standard practice for many of the major surgical procedures. The purpose of prophylactic antibiotics is to reduce and minimize the incidence of post-operative wound infections. Antibiotic consumption in humans is increasing globally. The greatest increase between 2000 and 2010 was in low- and middle-income countries, but in general, high-income countries still use more antibiotics per capita [1]. Growing economic prosperity and rising incomes, as well as expanding insurance coverage, have increased antibiotic consumption [2]. The countries consuming the most antibiotics overall in 2010 were India, 13 billion SU; China, 10 billion SU; and the United States, 7 billion SU [1]. Among hospital acquired infections, SSI accounts for $14-16 \%$ of the inpatient infections [3]. The achievement of a rational choice and appropriate use of antibiotics and to recognize their potential problems are the main challenges in the prescription of antibiotics. Detailed knowledge of pharmacological and microbiological factors and clinical judgment for the therapy of infectious diseases is required for the optimal and judicious selection of antimicrobial agents. Increased accesses to antibiotics have lowered morbidity and mortality and are also the driving force for antibiotic resistance.

The risk of SSI, the potential severity of the consequences of SSI, the effectiveness of prophylaxis in that operation, the consequences of prophylaxis for that patient is directly dependent on the decision regarding the benefits and risks of prophylaxis for an individual patient. Their imperceptive and non-judicious use increases the risk of bacterial drug resistance and thus has prompted the need to use antibiotics judiciously. In this study, we want to focus on the pattern of antibiotic utilization in surgical patient in our hospital.

\section{METHODS}

This is a hospital-based prospective observational study conducted on surgical patients for whom surgical procedures were done and have been admitted in Bharati Hospital and Research Centre, Pune. All patients who have been operated in the 6-month period (October 2015 to March 2016) were included in the study. After the approval of the Ethics Committee of Bharati Hospital Research Centre, the data were collected according to the inclusion criteria, i.e., patients above 18 years of age of both genders admitted for surgery. In total, 160 patients with surgical operations were included in the study.

Patient profile forms consisting of demography, etiology, history, diagnosis, antibiotic drug therapy of the patients was collected. Evaluation of the data of antibiotic utilization was done using suitable standard treatment guidelines such as Scottish Intercollegiate Guidelines Network (SIGN), American Society of Health-System Pharmacists (ASHP) guidelines, and Infectious Diseases Society of America (IDSA) guidelines.

\section{RESULTS}

A total of 160 patients admitted in surgery were investigated prospectively during 6-month period. Out of 160 patients, 108 were male patients and 52 were female (Table 1$) .35$ patients (21.87\%) were in the age group of 18-33 years, 52 patients (32.5\%) were in the age group of 34-49 years, 48 patients (30\%) were in the age group of 50-65 years while 25 patients $(15.62 \%)$ were in the group of 66-81 years of age (Table 2). 
About 160 cases were classified into respective system-wise surgical procedures of which $79(49.37 \%)$ cases are skin and soft tissue infections, 41 (25.62\%) cases of general surgical procedure, $20(12.5 \%)$ cases of gastrointestinal surgical procedure, $18(11.25 \%)$ cases of urinary system, and $2(1.25 \%)$ case of head-neck system (Fig. 1).

Out of the 160 patients that were included for the study, 33 (20.62\%) patients had hernia, 29 (18.12\%) patients had Cellulitis, 27 (16.87\%) patients had diabetic foot ulcer, $27(16.25 \%)$ patients had abscess, and 17 (10.62\%) patients had appendicitis and cholelithiasis (Table 3).

Furthermore in this study, it was found that 471 antibiotics were used in total of 160 patients, among which highest group of antibiotics prescribed were third generation cephalosporin. The prescribing pattern of the antimicrobials were penicillins - 111 (23.56\%), cephalosporins - 133 (28.23\%), nitroimidazoles - 94 (19.95\%), aminoglycosides - 41 (8.70\%), oxazolidine - 25 (5.30\%), fluoroquinolones - 35 (7.43\%), carbapenems - 11 (2.33\%), lincosamides and macrolides - 8 (1.69\%), respectively (Table 4 ). The most extensively prescribed drugs were the cephalosporin followed by penicillins. The most frequently prescribed antibiotics were metronidazole - 19.74\% among the nitroimidazoles followed by ceftriaxone $-19.53 \%$ of the class cephalosporins.

The total number of fixed drug combinations was 4, i.e., amoxicillin and clavulanate, piperacillin and tazobactum, ampicillin and cloxacillin, sulfamethoxazole and trimethoprim, out of which the most commonly used combination was amoxicillin and clavulanate - 64 (13.58\%).

Moreover, in this study, details of antibiotics which were prescribed the highest in a particular disease are as follows. Total 100 antibiotics $(21.23 \%)$ were administered in cellulites patients out of total of 471 antibiotics. Amoxicillin + clavulanic acid (5.94\%) is highly prescribed, whereas lincomycin is least prescribed in patients. Total 106 antibiotics $(22.50 \%)$ were administered in diabetic foot and ulcer patients out of total of 471 antibiotics. Ceftriaxone (4.03\%) is highly followed by linezolid (2.97\%) and metronidazole (3.60\%), where cefixime is least prescribed in patients. In hernia, total 62 antibiotics $(13.16 \%)$ were prescribed in patients of this study, ceftriaxone $(5.94 \%)$ is highly prescribed, whereas cefixime, ciprofloxacin, cephalexin are least prescribed in study. In appendicitis, $2.97 \%$ ceftriaxone among the antibiotics were prescribed and prescribing of metronidazole was $2.97 \%$ whereas piperacillin + tazobactum are the lowest. In abscess, total $84(17.83 \%)$ antibiotics were prescribed in patients. Amoxicillin + clavulanate $(4.03 \%)$ and metronidazole $(5.09 \%)$ are the highest, where linezolid, ciprofloxacin, meropenem, and ceftazidime are the least prescribed (Table 5).

Among all antibiotics, ceftriaxone (83.69\%) acquired the top preoperatively prescribed antibiotic and the next was metronidazole (43.01\%), amoxicillin + clavulanate (59.37\%), ciprofloxacin $(33.33 \%)$, etc. The top post-operative antibiotics prescribed were metronidazole (76.34\%), ceftriaxone (47.82\%), followed by and so on (Table 6 and Figs.2 and 3).

\section{DISCUSSION}

Surgical antibiotic prophylaxis is an adjunct to, not a substitute for good surgical technique. Antibiotic prophylaxis should be regarded as one of the components of an effective policy for the control of health care associated infection. Prophylactic administration of antibiotics inhibits the growth of contaminating bacteria and their adherence to prosthetic implants, thus reducing the risk of infections. Administration of antibiotics also increases the prevalence of antibiotic - resistant bacteria [4].

Patients in surgical ward develop infections post-surgery; many of the infections are caused by bacteria's that are highly virulent. The use of antibiotics in surgical patients both for prophylaxis and treatment
Table 1: Demographic details of patients

\begin{tabular}{ll}
\hline Sex & Number of patients (\%) \\
\hline Male & $108(67.5)$ \\
Female & $52(32.5)$ \\
\hline
\end{tabular}

Table 2: Age group categorization

\begin{tabular}{ll}
\hline Age & Number of patients (\%) \\
\hline $18-33$ & $35(21.87)$ \\
$34-49$ & $52(32.5)$ \\
$50-65$ & $48(30)$ \\
$66-81$ & $25(15.62)$ \\
\hline
\end{tabular}

Table 3: Disease pattern reported

\begin{tabular}{ll}
\hline Diagnosis & Number of patients (\%) \\
\hline Hernia & $33(20.62)$ \\
Cellulitis & $29(18.12)$ \\
Diabetic foot ulcer & $27(16.87)$ \\
Abscess & $26(16.25)$ \\
Appendicitis & $17(10.62)$ \\
Cholelithiasis & $17(10.62)$ \\
Others & $11(6.87)$ \\
\hline
\end{tabular}

Table 4: Prescribing pattern of antibiotics

\begin{tabular}{ll}
\hline Antibiotics & Number of antibiotics (\%) \\
\hline Cephalosporin & $133(28.23)$ \\
Penicillin & $111(23.56)$ \\
Nitroimidazoles & $94(19.95)$ \\
Aminoglycosides & $41(8.70)$ \\
Fluoroquinolones & $35(7.43)$ \\
Oxazolidine & $25(5.30)$ \\
Carbapenems & $11(2.33)$ \\
Lincosamides & $8(1.69)$ \\
Macrolides & $8(1.69)$ \\
Others & $5(1.06)$ \\
\hline
\end{tabular}

Table 5: Disease-wise high antibiotics

\begin{tabular}{lll}
\hline Disease & \multicolumn{1}{l}{ Highest antibiotics } \\
\cline { 2 - 3 } & Antibiotics & n (\%) \\
\hline Cellulitis & Amox+clavulanic acid & $28(5.94)$ \\
Diabetic foot and ulcer & Ceftriaxone & $19(4.03)$ \\
Abscess & Metronidazole & $24(5.09)$ \\
Cholelithiasis & Ampi+cloxacillin & $12(2.54)$ \\
Appendicitis & Metronidazole & $14(2.97)$ \\
Hernia & Ceftriaxone & $28(5.94)$ \\
Others & Metronidazole & $8(1.69)$ \\
\hline
\end{tabular}

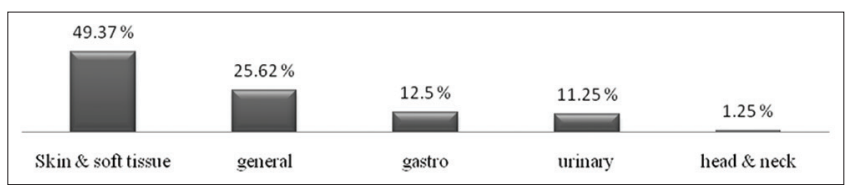

Fig. 1: System-wise disease pattern

of infections is a justifiable practice that however requires a regular review of the chosen regimen on the grounds of efficacy, diagnosis pattern, prescribing pattern and the aspects to maximize the benefits to the patients [5]. Antibiotics are among the most commonly prescribed drugs in hospitals and developed countries around $30 \%$ of the hospitalized patients are treated with antibiotics [6]. 
Table 6: Prescribing pattern of pre- and post-antibiotics

\begin{tabular}{|c|c|c|c|c|}
\hline Antibiotics & Pre-operative & $\%$ Pre-operative & Post-operative & $\%$ Post-operative \\
\hline Ceftriaxone & 77 & 83.69 & 44 & 47.82 \\
\hline Metronidazole & 40 & 43.01 & 71 & 76.34 \\
\hline Ciprofloxacin & 10 & 33.33 & 16 & 53.33 \\
\hline Amoxicillin+clavulanic acid & 38 & 59.37 & 48 & 75 \\
\hline Clindamycin & 2 & 25 & 6 & 75 \\
\hline Amoxicillin & 2 & 50 & 2 & 50 \\
\hline Ampicillin+cloxacillin & 8 & 50 & 10 & 62.5 \\
\hline Cefotaxime & 3 & 60 & 2 & 40 \\
\hline Piperacillin+tazobactum & 8 & 29.62 & 23 & 85.18 \\
\hline Gentamycin & - & - & 15 & 78.94 \\
\hline Linezolid & 2 & 8 & 15 & 60 \\
\hline Amikacin & 4 & 18.18 & 16 & 72.72 \\
\hline Azithromycin & - & - & 5 & 100 \\
\hline Cefixime & - & - & 5 & 100 \\
\hline Cephalexin & 2 & 100 & 2 & 100 \\
\hline Ceftazidime & - & - & 2 & 100 \\
\hline Doxycycline & 2 & 66.66 & 1 & 33.33 \\
\hline Faropenem & - & - & 3 & 60 \\
\hline Meropenem & - & - & 2 & 33.33 \\
\hline
\end{tabular}

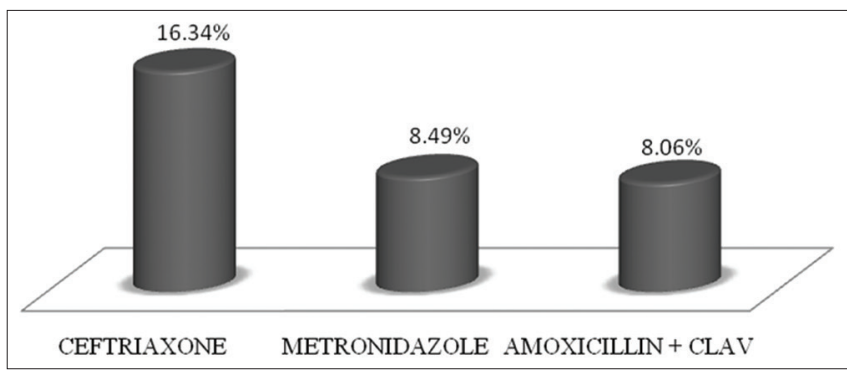

Fig. 2: Highest pre-operative antibiotics

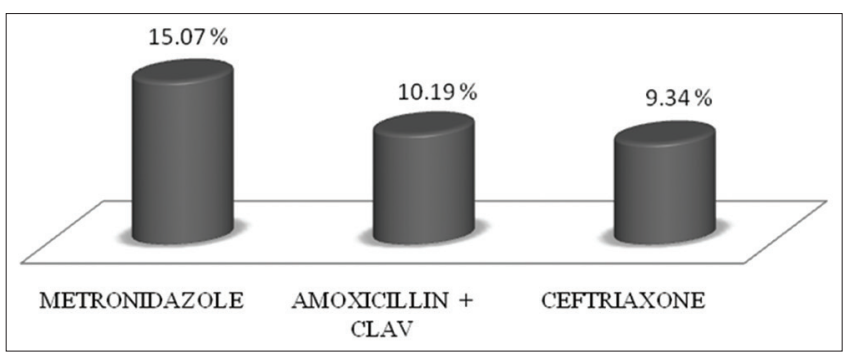

Fig. 3: Highest post-operative antibiotics

This prospective study attempts to assess the general pattern of how antibiotics are used in surgical wards which were conducted for the duration of 6 months, in which 160 patients were enrolled in accordance to the studies inclusion criteria. According to SIGN guidelines the goals of prophylactic administration of prophylactic administration of antibiotics to reduce the incidence of SSI, use of antibiotics in a manner that is supported by evidence of effectiveness, minimize the effect of antibiotics on the patients normal bacterial flora, minimize adverse effects and cause minimal change to the patients host defences [4].

In our study, among the total group of 160 patients there were $108(67.5 \%)$ male and $52(32.5 \%)$ female patients. From the total number of patients, the maximum number of patients operated for different surgeries were found to be 52, i.e., (32.5\%) lying under the age group of 34-49 years, and the minimum group of patients admitted were 25 , i.e., $(15.62 \%)$ of the age group above 66 years of age. Similar study conducted on pattern of antibiotic usage in surgical inpatients of a teaching hospital at Gondar College of Medical Sciences in Northwest
Ethiopia in 2004 which also had similar gender distribution, i.e., male patients are greater than female patients.

Moreover, in this study, all the cases were classified into their systemic surgical procedure, i.e., skin and soft tissue (includes of cellulites, diabetic foot ulcers, and abscess), general surgical procedures (includes hernia repair and colostomy closure), gastrointestinal tract surgical procedures (includes appendectomy), urinary surgical procedures (includes cholelithiasis), and head and neck system which includes goiter cases. Skin and soft tissue surgery procedures accounts for the highest number, i.e., $49.37 \%$ of cases, 41 , i.e., $25.62 \%$ of general surgical category, 20 cases, i.e., $12.5 \%$ of gastrointestinal surgical procedures, 18 cases, i.e., $11.25 \%$ of urinary systems, etc. Similar study was conducted on antibiotics prescribing pattern in surgical wards of department of surgery at Rajah Muthiah Medical College Hospital in Tamil Nadu in 2014 which also had the similar diagnosed pattern of cases, i.e., appendicitis $23.04 \%$, diabetic foot ulcers $29.78 \%$, and hernia $21.27 \%$. Some of other studies also have major account for appendicitis and hernia in India [7].

In this study, out of 160 patients the maximum number, i.e., 33 (20.62\%) patients had hernia and the minimum number, i.e., 11 (6.87\%) patients had other surgeries such as goiter, colostomy, and intestinal obstruction. Moreover, 27 (10.87\%) patients had diabetic foot ulcer, $29(18.12 \%)$ patients had cellulitis and $27(16.25 \%)$ patients had abscess. A similar study was found which showed contaminated surgeries accounts for $35.45 \% \quad(n=39)$ which includes wound debridement, incision and drainage, split skin graft, epigastric hernia repair and fissurectomy. Clean contaminated surgeries accounts for $63.37 \%(\mathrm{n}=45)$ which include appendectomy and cholecystectomy. Above study was conducted on antimicrobial prophylaxis pattern in surgical procedures and incidences of post-operative infection at Department of General Surgery, MVJMC and RH, Hoskote in Bengaluru in 2015 [8].

In this study, it was found that 471 antibiotics were used in total of 160 patients, among which highest group of antibiotics prescribed were third generation cephalosporin comprising $28.23 \%$ and $23.56 \%$ of penicillin's, and then $19.95 \%$ metronidazole. Hence, the prescribing pattern of antibiotics in the surgery ward was found to be aminoglycosides $8.70 \%$, fluoroquinolones $7.43 \%$, oxazolidine $5.30 \%$, lincosamides $1.69 \%$, carbapenems $2.33 \%$, and macrolides $1.69 \%$. In a study involving, the analysis of treatment records for 150 in patients at a primary health complex in Bangladesh in 2009, the highest prescribed 
antibiotic was ceftriaxone (30.19\%) followed by cefixime (18.87\%) and amoxicillin $(16.98 \%)$. This varies from the study due to difference of antibiotic pattern between inpatient and outpatient department. In hospitals injectable antibiotics are more used due to morbid condition of the patients [9]. Another study which was conducted in prescribing patterns of antibiotics in post-operative patients in teaching hospital in Hyderabad in 2014 which also had similar antibiotic prescribing pattern, among which highest group of antibiotics prescribed were third generation cephalosporin comprising $44 \%$ and then $30.7 \%$ metronidazole.

Another study conducted in tertiary care hospital of Pondicherry, South India which showed $(35.60 \%)$ cephalosporins and (21.30\%) penicillins were the most commonly prescribed groups of antibiotics [10]. Cephalosporin is a very important class of drugs which have been prescribed extensively as an antimicrobial in the treatment of acute and chronic bacterial infection. They have been very successful in treating and controlling infections. However, there are growing numbers of reports of resistance to these agents with increasing use. Cephalosporin's usage pattern exerts a significant influence over the rates of resistance observed and led to problematic multidrug resistant nosocomial pathogens [11]

In this study, our hospital has their own antibiotic policy for infectious and surgical prophylaxis which is similar to ASHP guidelines and IDSA guidelines. According to above guidelines for diabetic foot ulcer and cellulitis standard first line treatment is flucloxacillin + ciprofloxacillin and metronidazole where our hospital has recommended the use of cloxacillin or cefazolin or clindamycin. However, actual given therapy is ceftriaxone and metronidazole and linezolid or amoxicillin + clavulanic acid. Total 100 antibiotics (21.23\%) were administered in cellulites patients out of total 471 antibiotics. Amoxicillin + clavulanic acid $(5.94 \%)$ is highly prescribed in patients whereas total 106 antibiotics $(22.50 \%)$ were administered in diabetic foot ulcer patients. This ceftriaxone $(4.03 \%)$ is highly followed by linezolid $(2.97 \%)$ and metronidazole $(3.60 \%)$.

For appendicitis and abscess, the standard first line treatment according to the guidelines is Gentamycin, metronidazole and amoxicillin; and according to hospital policy ceftriaxone, metronidazole or piperacillin + tazobactum, and the actual antibiotics which were administered to patients are ceftriaxone, metronidazole, gentamycin or amoxicillin + clavulanic acids. In appendicitis, $2.97 \%$ ceftriaxone among the antibiotics were prescribed. In abscess, total 84 antibiotics $(17.83 \%)$ were prescribed among which $5.09 \%$ metronidazole was prescribed. For hernia, antibiotic which is standard, i.e., ceftriaxone is administered. In hernia, total 62 antibiotics $(13.16 \%)$ were prescribed in this study, ceftriaxone which is $5.94 \%$ highly prescribed.

This study also described about pre-operative and post-operative antibiotics for prophylaxis purpose. Among all antibiotics, ceftriaxone and metronidazole are prescribed as pre-operatively $83.69 \%$ and $43.01 \%$, respectively. Postoperatively $76.34 \%$ metronidazole and $47.82 \%$ ceftriaxone were prescribed. Average number of drugs per prescription is an important indicator of prescription audit. This is preferable to keep the mean number of drugs per prescription as minimum as possible. This will help to avoid the drug-drug interactions, development of bacterial resistance and increase hospital cost. In this study, the average number of antimicrobials prescribed in surgery department was 3 per patients. This is similar to that reported by Deshmukh, prescribing pattern of antimicrobial agents in indoor patients of SRTR Government Medical College, Maharashtra, which was 3.02 [12]. Another study conducted in Dr. B.R. Ambedkar Medical
College, Bengaluru in 2012, and drug utilization study of antimicrobials in post-operative showed that the average number of drugs prescribed was 2.5 \pm 3.02 which is similar to our study [13]. Another study conducted in Department of Community Health Sciences and Women and Child Health Division, Aga Khan University, Stadium Road, Karachi, Pakistan in 2013, and rational prescription and use showed that the average number of drugs prescribed was 3 or more which is similar to our study [14].

Appropriate antibiotic prophylaxis can reduce the risk of wound infections after surgery, but additional antibiotic use also increases the selective pressure favoring the emergence of antimicrobial resistance.

\section{CONCLUSION}

It is evident that the usage of antibiotics was found to be inappropriate in many conditions. The antibiotic prescribing was not found to be following the hospital antibiotic policy and any other standard treatment guideline. Third generation cephalosporin was the preferred or most prescribed choice of drug for prophylaxis followed by penicillins. The average number of antimicrobials prescribed in surgery department was 3 per patients. Amoxicillin and clavulanate were the fixed drug combination that was prescribed maximally.

\section{REFERENCES}

1. Centre for Disease Dynamics, Economics and Policy. State of the World's Antibiotics. Washington, DC: CDDEP; 2015.

2. Masiero G, Filippini M, Ferech M, Goossens H. Socioeconomic determinants of outpatient antibiotic use in Europe. Int J Public Health 2010; 55(5):469-78

3. Patherick ES, Dalton JE. Methods for identifying surgical wound infections after discharging from hospital: A systematic review. BMC Infect Dis 2006;6:170-8.

4. Scottish intercollegiate guidelines network 104, Antibiotic Prophylaxis in Surgery. A National Clinical Guideline. July; 2018 and updated April; 2014.

5. Abula T, Kedir M. The pattern of antibiotic usage in surgical in-patients of a teaching hospital, Northwest Ethiopia. Ethiop J Health Dev 2004; $18(1): 35-8$

6. George SS, Varghese SR, Samuel CJ. Evaluation of antibiotic prescribing patterns among medical practitioners in North India. Indian J Basic Appl Med Res 2013;8(2):952-7.

7. Kumar BA, Adiveni T, Chandra DS, Charan N, Padmini P, Ashwini K, et al. Study and use of antimicrobials in post-operative wound infections in gastrointestinal surgeries. Indo Am J Pharm Res 2013;3(4):1-5.

8. Babu KJ, Devarajan K, Paidi NR, Ramaswamy R, Sudarshan P. Study of antimicrobial prophylaxis pattern in surgical procedures and incidence of post-operative infection - A retrospective study. Indo Am J Pharm Res 2015;5(3):1-9.

9. Chowdhury MK, Siddique AA, Sarkar PK, Haque M. Pattern of antibiotic use in different departments of Dhaka medical college hospital. J Med 2015;16(1):35-8.

10. Pandiamunian J, Somasundaram G. A study on prescribing pattern of anti microbial agents in the medical intensive care unit of a tertiary care teaching hospital in Puducherry Union Territory, South India. Int J Pharm Pharm Sci 2014;6(3):235-8.

11. Kheder S. Cephalosporins usage and resistance trend in a sudanese hospital surgical wards. J Pharm Biomed Sci 2011;11(3):1-6.

12. Deshmukh VS, Khadke VV, Patil AW, Lohar PS. Study of prescribing pattern of antimicrobial agents in indoor patients of a tertiary care hospital. Int J Basic Clin Pharmacol 2013;2(3):229-36.

13. Patil S, Padma L, Veena DR, Shanmukananda P. Drug utilization study of antimicrobials in post-operative wards in a teaching hospital. Int Res J Pharm Appl Sci 2012;2(5):56-9.

14. Zaidi S, Nishtar NA. Rational prescription and use: A snapshot of the evidence from Pakistan and emerging concerns. Int J Pharm Pharm Sci 2013;5(1):131-5. 